\title{
DISKUSSION
}

DDS - Die Deutsche Schule

112. Jahrgang 2020, Heft 3, S. 338-353

https://doi.org/10.31244/dds.2020.03.09

(c) 2020 Waxmann

Christian Timo Zenke

\section{„Vom Klassenzimmer zur Lernlandschaft“? \\ Über eine Expedition ins Ungewisse}

\section{Zusammenfassung}

Der Artikel widmet sich aktuellen Entwicklungen im Bereich der Schulraumgestaltung sowie daraus resultierenden Herausforderungen für Erziehungswissenschaft, Schulentwicklung, Lehrerbildung und Politik. Unter Bezugnahme speziell auf internationale Veröffentlichungen zum Thema wird dabei die Notwendigkeit a) einer systematischen Evaluation alternativer Schulraummodelle sowie b) einer gezielten Entwicklung von Strategien der raumbezogenen Lehrerprofessionalisierung diskutiert.

Schlüsselwörter: Schulentwicklung, Lehrerbildung, Schularchitektur, Schulraumgestaltung

\section{"From Classroom to Learning Landscape"?}

On an Expedition into the Uncertain

\section{Abstract}

This article focuses on current developments in the field of school-building layout and design and the resulting challenges for educational science, school development, teacher training, and politics. With special reference to international publications on the topic, it discusses the necessity of a) a systematic evaluation of alternative schoolroom designs as well as $b$ ) the continued development of teacher training that takes the layout of the learning environment into consideration.

Keywords: school development, teacher training, school architecture, school room design

\section{1 „Sanierungsstau“ als pädagogische Chance?}

Forderungen nach einer „zukunftsfähigen“ Gestaltung von Schulbauten, nach einer stärkeren Nutzerbeteiligung bei der Realisierung schulischer Bauprojekte sowie 
nach einer systematischeren Berücksichtigung des konstitutiven Zusammenhangs von (Schul-)Raum und Pädagogik erleben derzeitig einen regelrechten Boom: Nicht nur ist das Auftragsvolumen zur Errichtung neuer Schulgebäude in den vergangenen Jahren massiv gestiegen; auch Erziehungswissenschaft, Schulpädagogik und Architektur widmen sich intensiver denn je der Frage, wie eine angemessene "Schule des 21. Jahrhunderts" (Ramseger, 2017, S. 6) auch auf baulicher Ebene aussehen könnte (vgl. bspw. Auer \& Nagler, 2017; Kahlert, Nitsche \& Zierer, 2013; Montag Stiftung Jugend und Gesellschaft, 2017; Stadler-Altmann, 2016b). Dasselbe gilt für die allgemeine Medienlandschaft: Warnungen vor einer "Schulbaukrise“ (Windrath, 2019, S. 30) und einem zunehmenden „Verrotten“ unserer Schulen (Brost, 2015) werden ebenso regelmäßig ausgerufen wie die Hoffnung darauf, durch neue Schulbaukonzepte zugleich auch "neue, offenere Unterrichtsformen“ zu ermöglichen (Wetzel, 2019, S. 22).

Sucht man nach den Gründen für diese Entwicklung, so stößt man dabei zunächst einmal auf einen vorwiegend bautechnischen Aspekt, der mit der durchschnittlichen Nutzungsdauer öffentlicher Gebäude zu tun hat: So wurden im deutschsprachigen Raum gerade in den späten 1960er- und frühen 1970er-Jahren zahlreiche Schulen neu gebaut (unter anderem als Reaktion auf „Babyboomer“ und „Bildungskatastrophe“), die jedoch über viele Jahre nicht ausreichend renoviert wurden und nun einen massiven Sanierungsstau erzeugen (vgl. Hedtke, 2016; Schneider, 2012). Auf diesen Umstand wiederum reagieren Bund und Länder seit einigen Jahren mit einer rasanten Erhöhung ihrer entsprechenden Etats für Schul- und Bildungsbauten: So plant allein das Bundesland Berlin, in den kommenden Jahren 2,8 Milliarden Euro in den Bau neuer Schulen zu investieren (vgl. Wetzel, 2019, S. 22), während die Bundesregierung jüngst ein fünfähriges Programm zur Verbesserung der Schulinfrastruktur finanzschwacher Kommunen mit einem Gesamtetat von 3,5 Milliarden Euro aufgelegt hat (Bundesministerium der Finanzen, 2019) und für den gesamten deutschsprachigen Raum bis 2030 zuweilen gar mit Investitionen von mehr als 67 Milliarden Euro im Bereich der Schul- und Bildungsbauten gerechnet wird (vgl. Puls, o. J.).

Gleichzeitig jedoch ist ebenjener „Boom“ auch eng mit pädagogischen und schulentwicklungstheoretischen Entwicklungen verknüpft. Dabei sind es neben veränderten Raumbedarfen durch Ganztagsschulentwicklung (vgl. Reich, 2012) und Digitalisierung (vgl. Montag Stiftung Jugend und Gesellschaft, 2017, S. $46 \mathrm{ff}$.) sowie einer zunehmenden Konzentration von Bildungspolitik und Erziehungswissenschaft auf die Einzelschule als lernende Institution (vgl. Zenke, 2018a) insbesondere Fragen der Inklusion, die die gängige Schulbau-Praxis verstärkt mit neuen Herausforderungen konfrontieren. So wurde spätestens mit der Ratifizierung der UNKonvention über die Rechte von Menschen mit Behinderungen eine Entwicklungsaufgabe an die deutsche Schullandschaft herangetragen, deren Auswirkungen auch auf Belange der Schulraumgestaltung kaum zu übersehen sind: „Inklusive“ Schulen brauchen demnach, wie die Diskussion der vergangenen Jahre gezeigt hat, nicht 
nur barrierefreie Räumlichkeiten und Verkehrswege, sondern darüber hinaus schulische Raumarrangements, die ein gemeinsames, individualisiertes Lernen in heterogenen Gruppen befördern - und zwar bei gleichzeitiger Anwesenheit verschiedener Pädagog*innen (vgl. Brokamp \& Platte, 2011; Kricke, Reich, Schanz \& Schneider, 2018; Schöning \& Schmidtlein-Mauderer, 2015; Zenke, 2017). Damit allerdings werden zugleich bauliche Lösungen notwendig, die allein schon wegen der „zeitlich divergierenden interindividuellen Lernbedürfnisse der Heranwachsenden“ sowohl „kurzfristige als auch langfristige Veränderungen zulassen“ (Schöning \& SchmidtleinMauderer, 2016, S. 87) - und sich dementsprechend vom bisherigen Modell der Klassenraum-Flur-Schule zu lösen beginnen.

Ganz in diesem Sinne wird in der aktuellen Diskussion zum Thema denn auch immer wieder für eine bauliche wie pädagogische Wende „vom Klassenzimmer zur Lernlandschaft" plädiert (vgl. bspw. Reich, 2014, S. 219): für ein grundlegendes Neudenken schulischer Raumstrukturen also, das den skizzierten „Sanierungsstau“ als Ausgangspunkt nutzt für eine nachhaltige, gerade auch pädagogische Veränderung schulischen Lehrens und Lernens (siehe hierzu genauer Zenke, 2018a). In diesem Zusammenhang sind es insbesondere zwei Schulraummodelle, die immer wieder als besonders geeignete Raumarrangements eines gerade auch inklusiven Lernens und Lehrens in der Schule genannt und in einigen Fällen auch tatsächlich bereits realisiert werden: das Prinzip des „Lern-Clusters“ und dasjenige der „Offenen Lernlandschaft“ (vgl. etwa Imhäuser, 2012, S. 193 ff.; Montag Stiftung Urbane Räume, Montag Stiftung Jugend und Gesellschaft, Bund Deutscher Architekten \& Verband Bildung und Erziehung, 2013, S. $26 \mathrm{ff}$; Kricke et al., 2018, S. $450 \mathrm{ff}$.). ${ }^{1}$ Beide Konzepte sind dabei zwar auf eine „variable Nutzung der Räume“ (Montag Stiftung Urbane Räume et al., 2013, S. 26) hin ausgelegt, sie unterscheiden sich allerdings erheblich in der konkreten baulichen Umsetzung dieses Prinzips: Während das Modell des „Lernclusters“ gekennzeichnet ist durch eine "große Vielfalt unterschiedlicher Raumsituationen in einem definierten Teilbereich des Schulgebäudes“ (ebd., S. 27), löst sich die „Offene Lernlandschaft“ nahezu komplett vom „herkömmlichen Verständnis eines allgemeinen, nach Klassenräumen gegliederten Lern- und Unterrichtsbereichs“ (ebd., S. 28) und bietet den Nutzer*innen anstelle dessen „die Auswahl zwischen unterschiedlichen Lernbereichen und -atmosphären“ (ebd.): vom einzelnen „Think-Tank“ über nutzungsvariable Großraumbereiche bis hin zu Auditorien und Außenanlagen.

1 Als drittes Schulraummodell wird darüber hinaus immer wieder der sogenannte „Klassenraum-Plus" genannt, der den Bedarf nach "multioptional nutzbaren Lern- und Unterrichtsbereichen“ durch eine „Vergrößerung, Verknüpfung oder veränderte Zonierung von Basis- oder Klassenräumen" zu erfüllen versucht (Montag Stiftung Urbane Räume et al., 2013, S. 27). Da dieses Modell allerdings, wie Kricke et al. (2018) es formulieren, „nicht nur baulich, sondern auch pädagogisch an vielen Stellen an Bekanntes anknüpft", beinhaltet es zugleich das Risiko, „dass trotz baulicher Veränderungen am tradierten Schulmodell (z. B. Zwei-Gruppen-Setting) festgehalten wird, in dem ohne veränderte pädagogische Strukturen Inklusion scheitert" (S. 485). 


\section{Die Großraumschulen der 1970er-Jahre als Warnung}

Bei aller Euphorie jedoch, die in der diesbezüglichen Diskussion immer wieder mitschwingt, droht schnell aus dem Blick zu geraten, dass ganz ähnliche Hoffnungen und Lösungsvorschläge bereits vor mehr als fünfzig Jahren formuliert worden waren - und im Anschluss weitestgehend scheiterten. So wurde insbesondere in den späten 1960er- und frühen 1970er-Jahren ebenfalls intensiv für eine verstärkte Öffnung und Flexibilisierung schulischer Raumstrukturen plädiert und unter dem Schlagwort „Großraumschule“ schließlich auch zunehmend mit entsprechend „offenen“ Schulraummodellen in der Praxis experimentiert (etwa in Fröndenberg, Osterburken, Weinheim und Rodenkirchen). Mit Ausnahme weniger Einzelfälle jedoch (namentlich der beiden Bielefelder Schulprojekte Laborschule und OberstufenKolleg) gelang es keinem der damals realisierten Schulbauexperimente, die in ihre veränderte Raumstruktur gesetzten pädagogischen Hoffnungen tatsächlich nachhaltig zu erfüllen, weshalb die meisten jener Schulen denn auch bereits wenige Jahre nach ihrer Eröffnung wieder so stark zurückgebaut oder gar abgerissen wurden, dass sie aus heutiger Sicht kaum mehr als Großraumschulen oder gar Lernlandschaften zu erkennen sind (vgl. hierzu sowie zur bedauerlicherweise noch viel zu wenig beforschten Geschichte deutscher Großraumschulen: Blömer, 2011; Huber \& Thormann, 2002; Zenke, 2019; Zinner, 2014).

Während das aus dieser Parallele entstehende Spannungsverhältnis zwischen (heutigem) Anspruch und (historischer) Wirklichkeit allerdings hierzulande bisher kaum thematisiert wird, gibt es im englischsprachigen Raum bereits seit mehreren Jahren eine intensive Debatte darüber, welche Lehren aus dem auch international zu konstatierenden „demise of open plan classrooms“ (Imms, 2016, S. 27) der 1970er-Jahre für die Entwicklung heutiger Innovative Learning Environments (ILE) ${ }^{2}$ gezogen werden könnten (vgl. bspw. Deed \& Lesko, 2015; Imms, 2016, 2018; Lippman \& Matthews, 2018). So resümiert etwa Imms (2018) mit Blick insbesondere auf Australien und die USA, ,the failure of various governments' 1970s attempts to modernise learning and teaching through the introduction of open plan schools" sei insbesondere auf zwei Gründe zurückzuführen: „first, poor evaluation; and second, the poor preparedness of teachers for these spaces." (S. 108) Und er ergänzt:

2 Andere, im englischsprachigen Raum ebenfalls (wenn auch deutlich seltener) verwendete Bezeichnungen für die gemeinten Formen der Schulraumgestaltung sind New Generation Learning Environments (NGLE), Modern Learning Environments (MLE) sowie Learning Landscapes. Siehe hierzu sowie zu einer damit verbundenen Arbeitsdefinition speziell der Bezeichnung Innovative Learning Environments genauer Alterator \& Deed, 2018, S. 5 f.; zu einer Typologie entsprechender Raumformen siehe darüber hinaus Imms, Cleveland \& Fisher, 2016. (Hinweis: Die hier gemeinte Verwendung des Begriffs Innovative Learning Environments ist nicht identisch mit derjenigen im Rahmen des gleichnamigen, von 2008 bis 2015 gelaufenen OECD-Projekts „Innovative Learning Environments“ (vgl. zu diesem Projekt u. a. Schrittesser, Fraundorfer \& Krainz-Dürr, 2012)). 
"This constitutes recognition that the evolution of ILEs is not a fad, but part of a long and steady process of improving education, one that has been in progress for hundreds of years. To continue this positive trajectory, for ILEs to be implemented successfully in the 2010s, we need rigorous evidence of their pedagogic performance in order to build strategies that skill teachers on their use." (Ebd.)

Ebendiese beiden von Imms hervorgehoben Erfordernisse a) der systematischen Evaluation der "pedagogic performance“ alternativer Schulraummodelle sowie b) der Entwicklung von „strategies that skill teachers on their use“ sollen im Folgenden daher denn auch etwas genauer in den Blick genommen werden - mit Fokus insbesondere auf die internationale Debatte zum Thema -, um im Anschluss sodann mögliche Konsequenzen gerade auch für die deutsche Schullandschaft und deren möglichen Weg „Vom Klassenzimmer zur Lernlandschaft“ diskutieren zu können.

\section{Alternative Schulraummodelle und ihre Erforschung}

Obwohl sich spätestens seit Beginn der 2010er-Jahre eine zunehmende Aufmerksamkeit auch der deutschsprachigen Erziehungswissenschaft für die pädagogische Dimension des Raumes beobachten lässt (vgl. Gebhard, Hummrich, Rabenstein \& Reh, 2015; Glaser, Koller, Thole \& Krumme, 2018; Kessl, 2016), liegen hierzulande bisher noch kaum Studien vor, die die konkrete Nutzung und pädagogische Wirksamkeit schulischer Räumlichkeiten dezidiert aus empirischer Perspektive in den Blick nehmen (vgl. zu diesem Desiderat auch Egger, 2019, S. 25; Stadler-Altmann, 2016a). Vielmehr werden, wie Stadler-Altmann (2016c) es formuliert, zumeist „pädagogische Überlegungen im Sinne von Bildungszielen und -entwürfen zum Ausgangspunkt genommen und über die Gestaltung und Nutzung von Klassenzimmern nachgedacht“ - wobei die „Nutzung des Klassenzimmers [...] als Bestätigung ihrer zuvor versprachlichten didaktischen Orientierungen und pädagogischen Überzeugungen gesehen“" wird (S. 11). Finden sich darüber hinaus schließlich doch vereinzelt empirische Arbeiten zum Thema (wie etwa Breidenstein \& Dorow, 2015; Egger, 2019; Zschiesche, 2019), so konzentrieren sich diese in der Regel auf verhältnismäßig traditionelle Klassenraum-Flur-Konzepte, während alternative Schulraummodelle wie Lerncluster-Schulen oder Offene Lernlandschaften kaum je explizit in den Blick genommen werden. ${ }^{3}$ Dies allerdings hat zugleich zur Folge, dass

3 Eine Ausnahme hiervon bildet im deutschsprachigen Raum neben den Arbeiten von Rabenstein, Reh, Kolbe et al. im Kontext des Projekts „Lernkultur und Unterrichtsentwicklung an Ganztagsschulen" (vgl. unter anderem Rabenstein \& Reh, 2008; Reh, 2011; Reh \& Kolbe, 2009, sowie unten, Fußnote 5) lediglich die Studie Raum und Inklusion von Kricke et al. (2018). Auch diese jedoch kann ihren selbstgesetzten Anspruch, im Rahmen einer „empirischen Studie“ zu untersuchen, „wie Inklusion [...] umgesetzt wird“ und was auf räumlich-pädagogischer Ebene "Gelingensbedingungen oder Stolpersteine auf diesem Weg sind“ (S. 31), nur begrenzt einlösen. Dies gilt dabei insbesondere für das Modell der „Lernlandschaft“: So wird dieses in den abschließenden „Empfehlungen aus dem Forschungsvorhaben“ zwar einerseits als besonders geeignetes Raumarrangement inklusiven Lehrens und 
bis heute weitestgehend ungeklärt ist, ob die genannten Modelle tatsächlich in der Lage sind, die diversen, immer wieder in sie gesetzten pädagogischen wie organisatorischen Hoffnungen auch tatsächlich zu erfüllen.

Ähnliches gilt für die internationale Forschung zum Thema: Zwar finden sich insbesondere im englischsprachigen Raum bereits deutlich mehr Arbeiten, die sich explizit (und in der Regel auch mit durchaus vielversprechenden Ergebnissen) der Nutzung und "pedagogic performance“ speziell von Innovative Learning Environments widmen (vgl. bspw. Deed \& Lesko, 2015; Gislason, 2010; Kariippanon, Cliff, Lancaster, Okely \& Parrish, 2018; Sigurðardóttir \& Hjartarson, 2016, sowie für einen entsprechenden Forschungsüberblick Acton, Riddle \& Sellers, 2018; Cleveland \& Fisher, 2014; Imms, 2018); insgesamt jedoch bleiben die damit verbundenen Evaluationsbemühungen, wie Gislason (2018, S. 198) anmerkt, bis heute ,an undeveloped field“. Ebendies hervorhebend konstatieren denn auch Acton, Riddle \& Sellers (2018, S. 205), es lägen bisher kaum belastbare empirische Erkenntnisse bezüglich der „sustained impacts of changed learning spaces on practice, social relationships and student outcomes" vor, und Cleveland (2016) schließlich resümiert ganz grundsätzlich mit Blick auf Fragen der ,evaluation of physical learning environments“:

"Approaches to evaluations that attempt to assess the effectiveness of physical learning environments in supporting pedagogical activities are in their infancy and require further development. [...] More research is required to develop rigorous methodologies and methods that can be confidently employed to assess the effectiveness of physical learning environments in supporting desired teaching and learning practices, activities and behaviors." (S. 94)

Es fehlt in diesem Sinne also nicht allein an belastbaren empirischen Erkenntnissen zum Thema; es scheint darüber hinaus auch methodologisch bei weitem nicht geklärt, wie die Nutzung und pädagogische Wirksamkeit von Schulräumen im Allgemeinen und alternativen Schulraummodellen im Besonderen am besten erforscht werden könnte. So gibt es zwar durchaus Konzepte der Post Occupancy Evaluation (POE), die - aus dem Bereich der Bau- und Stadtplanung stammend - darauf abzielen, die „Performance“ von Gebäuden im Anschluss an ihre Inbetriebnahme zu überprüfen (vgl. Alterator \& Deed, 2018; Cleveland, 2016; Cleveland \& Fisher, 2014); ebendiese Konzepte allerdings können, wie jüngst unter anderem Gislason (2018) und Wood (2018) gezeigt haben, nicht bruchlos auf den Bereich der Schulraumnutzung über-

Lernens hervorgehoben (vgl. S. 490 ff.); gleichzeitig jedoch konstatieren die Autor*innen, man habe in die als Grundlage für dieses Urteil dienende empirische Untersuchung leider „keine Schule mit einer ausgewiesenen Lernlandschaft aufnehmen können“, da solche „im deutschsprachigen Raum noch selten anzutreffen" seien (S. 492). Auch hier also überlagert die Erwartung gewissermaßen die Empirie: Obwohl keine "ausgewiesene“ Lernlandschaft Gegenstand der Untersuchung war, wird deren Prinzip - neben demjenigen des „Lernclusters“ - dennoch „langfristig und insbesondere bei Neubauten oder aufwändigen Umbauten“ als „Maßgabe“ einer „erfolgreichen inklusiven Zukunft“ (S. 500) beschrieben. 
tragen werden. Spätestens dann nämlich, wenn man Schulen als komplexe, lernende Institutionen begreift, denen als „democratic spaces“ zugleich Aufgaben zukommen, die weit über die Optimierung von reinen Lehr-Lernprozessen hinausgehen (vgl. Wood, 2018, S. 133), wird deutlich, dass die klassischen, zumeist quantitativ ausgerichteten Instrumentarien der POE zu kurz greifen:

"Post-occupancy evaluations [...] of educational facilities typically focus on
physical elements, such as heating, lighting, and acoustics, and occasionally include
some analysis of student-teacher interactions and environmental perceptions. [...]
However, evaluating ILEs involves another layer of complexity, partly because of the
diversity of innovative programs and their associated architectural requirements,
and partly because of the substantial qualitative research needed to determine how
the built environment is viewed by a school's occupant." (Gislason, 2018, S. 198)

Vor diesem Hintergrund wird seit einigen Jahren denn auch verstärkt dafür plädiert, zum Zwecke einer angemesseneren Evaluation gerade auch alternativer Schulraummodelle speziell auf praxistheoretische Zugänge zu setzen - und damit zugleich auf Instrumentarien der Teilnehmenden Beobachtung, der Videographie oder der Ethnographie (vgl. bspw. Bradbeer, 2016; Healy, 2016; Lippman \& Matthews, 2018). Auf diesem Wege, so die mit einem solchen Ansatz verbundene Hoffnung, könne es schließlich auch besser gelingen, dem relationalen Charakter pädagogischer Räume gerecht zu werden: dem Umstand also, dass jeder Raum - und damit auch jeder pädagogische Raum - „nie nur eine Substanz und nie nur eine Beziehung“ ist, „sondern aus der (An)Ordnung, das heißt aus der Plazierung in Relation zu anderen Plazierungen, entsteht“ (Löw, 2012, S. 224). ${ }^{4}$

Folgt man dieser Herangehensweise, so müsste es daher auch bei der Evaluation alternativer Schulraummodelle weniger um die Betrachtung schulischer Räume als „containers within which learning activities occur“ (Cleveland \& Fisher, 2014, S. 23) gehen, sondern vielmehr um die gezielte Erforschung von Praktiken des Hervorbringens pädagogischer Räume im schulischen Alltag. ${ }^{5}$ Andernfalls, so ließe sich angesichts der skizzierten Diskussion insbesondere zum Thema Post Occupancy Evaluation konsta-

4 Zum grundsätzlichen Zusammenhang von (erziehungswissenschaftlicher) Raumforschung und Praxistheorie siehe darüber hinaus auch Berdelmann \& Reh, 2015; Breidenstein \& Dorow, 2015; Göbel, 2016; Kalthoff, Rieger-Ladich \& Alkemeyer, 2015.

5 Als Ausgangspunkt für ein ebensolches Vorhaben könnten dabei im deutschsprachigen Raum unter anderem die bereits vorliegenden Arbeiten von Breidenstein, Dorow et al. sowie Rabenstein, Reh, Kolbe et al. dienen - weisen diese doch nicht nur auf die Fruchtbarkeit einer praxistheoretischen Annäherung speziell an das Zusammenspiel von Raum und Individualisierung hin (vgl. Breidenstein \& Dorow, 2015, sowie Breidenstein \& Rademacher, 2017), sondern darüber hinaus zugleich auf den Umstand, dass auch „neue Räume, die neue schulische Praktiken ermöglichen und teilweise erforderlich machen [...], von Normen durchdrungen“ sind und zugleich produziert sind von „Verboten und Anforderungen“: davon also, „was erwünscht und erlaubt ist und was man [...] an welchen Plätzen tun kann“ (vgl. Reh \& Kolbe, 2009, S. 115, sowie zu weiteren Ergebnissen aus demselben Projektzusammenhang auch Rabenstein \& Reh, 2008, sowie Reh, 2011). 
tieren, würde ebenjene von Imms eingeforderte Evaluation der „pedagogic performance" (Imms, 2018, S. 108) alternativer Schulraummodelle gerade die für pädagogische Räume so konstitutive „complex interrelationship between practice, pedagogical intent and space" (Acton et al., 2018, S. 206) aus dem Blick zu verlieren drohen und damit zugleich in die Falle einer "reduzierten Materialitätsperspektive“ geraten, die den „pädagogischen Ort auf seine bauliche oder territoriale, also seine formale Dimensionierung reduziert" (Kessl, 2016, S. 14).

\section{Lehrerbildung und „spatial literacy“}

Bereits der Begriff der Post Occupancy Evaluation allerdings zeigt darüber hinaus zugleich noch eine weitere Problematik an - impliziert er doch, dass die occupation eines Raumes (also dessen „Inbesitznahme“) ein vorübergehender, abschließbarer und zeitlich klar zu begrenzender Prozess sei, auf den dann eine wie auch immer geartete Post-Occupancy-Phase folgen könne. Dieser Annahme jedoch ließe sich - zumindest bezogen auf den Bereich der Schule - mit Bradbeer (2016) entgegnen, dass der Umzug in ein neues Schulgebäude vielmehr als „finished beginning“ (S. 76) verstanden werden sollte: also als ,starting point from which adaptations that support successful learning can occur" (S. 76 f.). Ganz in diesem Sinne schlägt denn auch Imms (2016, S. 32) vor, anstelle von „occupation“ lieber von „inhabitation“ zu sprechen: also davon „what happens over time, as compared to simply moving in“. Und er ergänzt: „Habitués actively and consciously manipulate their environment to pursue a set of scholastic and personal needs that often have little to do with what was planned.“ (Ebd.) Das allerdings bedeutet zugleich, dass es auch im Zuge einer erziehungswissenschaftlichen Auseinandersetzung mit alternativen Schulraummodellen weniger darum gehen sollte, einmal „okkupierte“ Schulräume zu evaluieren, um dann zu entscheiden, ob diese wie gewünscht „funktionieren“, sondern vielmehr darum, die konkreten Prozesse sowohl der occupation („moving in“) als auch der inhabitation („what happens over time“) selbst empirisch in den Blick zu nehmen (vgl. hierzu auch Berdelmann et al., 2016, insb. S. 83 ff.).

Ein aus einem ebensolchen Vorgehen resultierendes „,better understanding of the way that teachers occupy space“ (Bradbeer, 2016, S. 75) hätte dabei allerdings nicht allein den Vorteil, die tatsächliche Nutzung und Adaptation neuartiger Schulgebäude realistischer bewerten zu können; es könnte darüber hinaus zugleich als Grundlage dienen für die Entwicklung von Strategien, unter Zuhilfenahme derer Lehrerinnen während der genannten Phasen gezielt unterstützt werden könnten. Denn gerade dies zeigen die vorliegenden Studien zur Nutzung und „Inbesitznahme“ alternativer Schulraummodelle in aller Deutlichkeit (vgl. bspw. Gislason, 2018; Kariippanon et al., 2018; Sigurðardóttir \& Hjartarson, 2016; Zenke, 2019): Mag den untersuchten Gebäuden noch so sehr das Potenzial innewohnen, Prozesse insbesondere der 
Individualisierung sowie des Peer-Teaching zu unterstützen - die Frage, ob diese Potenziale in der Praxis auch tatsächlich abgerufen werden können, hängt ganz grundsätzlich und unmittelbar mit der „preparedness of teachers for these spaces“ (Imms, 2018, S. 108) zusammen. Oder, wie Deed \& Lesko (2015) es formulieren:

"While openness can be abstractly expressed through school architecture, the realisation of these authorisations is the result of teachers' thinking, practice and pedagogical engagement with the possibilities inherent within learning environments [...]. It is the individual teacher who must break with convention in order to take and apply the meaning of openness." (S. 219)

Ganz in diesem Sinne wird in der englischsprachigen Diskussion zum Thema denn auch immer wieder für eine gezielte Förderung der „spatial literacy“ von Lehrer*innen plädiert (vgl. Bradbeer, 2016, S. 79; Fisher, 2004, S. 37; Imms et al., 2016, S. 7): dafür also, diese systematisch auf den Umzug in ein neues, offeneres Schulgebäude vorzubereiten und sie dabei zugleich ganz grundsätzlich für die pädagogische Bedeutung des Raumes in der Schule zu sensibilisieren. Gislason (2018) etwa hebt dementsprechend denn auch explizit hervor, dass es gerade die diversen, mit einem ebensolchen Umzug verbundenen Anforderungen an „teacher training and curriculum development prior to occupancy“ (S. 187) seien, die als größte organisatorische Herausforderungen beim Wechsel von klassischen Schulräumen zu Innovative Learning Environments verstanden werden müssten - und er präzisiert:

"Teachers should be trained before they make the move to an ILE so they do not have to grapple with unconventional teaching methods while adapting to a new environment. Considerable lead time - ideally two to three years - should be devoted to curriculum development as well, so there is enough educational material ready when the program is launched and teachers are not caught short. Building a strong organizational and curricular foundation before occupancy is critical; failing to do so usually results in weak program implementation and increased teacher stress." (S. 187)

Die derzeitige Suche nach einem „zukunftsfähigen Schulbau“ gerät insofern in besonderem Maße auch zu einer Angelegenheit der Schul- und Unterrichtsentwicklung sowie der Lehrerbildung: Gerade ihnen kommt der Auftrag zu, organisatorische, pädagogische und didaktische Gelingens- wie Misslingensbedingungen der oссираtion und inhabitation alternativer Schulraummodelle $\mathrm{zu}$ evaluieren und (ausgehend von den dabei generierten Ergebnissen) Strategien $\mathrm{zu}$ entwickeln, wie der einzelnen Lehrkraft dabei geholfen werden könnte, das pädagogische Potenzial alternativer Schulraummodelle auch tatsächlich abzurufen und produktiv für den eigenen Schulund Unterrichtsalltag zu nutzen.

Da die Bearbeitung ebendieses Auftrags allerdings bis heute noch weitestgehend aussteht, bleibt gleichzeitig die Gefahr bestehen, dass die Versäumnisse der 1970er-Jah- 
re in Sachen Schulbauentwicklung in ganz ähnlicher Weise wiederholt werden. Denn: Zwar mögen heutige Lernlandschaften und Lerncluster-Schulen heller, freundlicher und abwechslungsreicher gestaltet sein als ihre Pendants der 1970er-Jahre (vgl. Zinner, 2014) - misslingt es jedoch erneut, deren erwartetes pädagogisches Potenzial auch tatsächlich in der Praxis fruchtbar zu machen, droht die eingangs skizzierte Hoffnung, den aktuellen „Sanierungsstau“ als Ausgangspunkt für eine nachhaltige, gerade auch pädagogische Veränderung schulischen Lehrens und Lernens zu nutzen, ein weiteres Mal - und damit möglicherweise endgültig - zu scheitern.

Dies gilt dabei in besonderem Maße auch für den deutschsprachigen Raum, in dem seit einigen Jahren zwar vermehrt (zumeist unter dem Stichwort „Phase Null“) Nutzerbeteiligungen zumindest bei der Planung von Schulbauten durchgeführt werden (vgl. hierzu genauer Berdelmann et al., 2016; Montag Stiftung Jugend und Gesellschaft, 2015, 2017; Seydel, 2016; Zenke, 2018b), es jedoch noch immer keinerlei elaborierte Konzepte gibt, an denen sich daran anschließende Bemühungen um eine pädagogische Begleitung auch der occupation und inhabitation jener neu errichteten Schulgebäude orientieren könnten. ${ }^{6}$

\section{5 „Into the Great Wide Open“?}

Der bereits im Titel dieses Aufsatzes anvisierte Weg „vom Klassenzimmer zur Lernlandschaft“ erweist sich rückblickend insofern nicht allein als eine „Expedition ins Ungewisse“, sondern darüber hinaus zugleich als ungemein steinig und hindernisreich. Möchte man vor diesem Hintergrund daher einerseits das von verschiedener Seite aus immer wieder aufgerufene (und in ersten Studien bereits angedeutete) pädagogische Potenzial alternativer Schulraummodelle auch im Deutschland der Jetztzeit tatsächlich produktiv für Zwecke der Schul- und Unterrichtsentwicklung nutzen und andererseits verhindern, dass in naher Zukunft Milliardenbeträge für den Bau von scheinbar innovativen Schulgebäuden ausgegeben werden, die sich im Anschluss sodann als inkompatibel mit den tatsächlichen Herausforderungen schulischer Praxis erweisen, braucht es in den nächsten Jahren eine gemeinsame, zielgerichtete Anstrengung von Wissenschaft, Schule und Politik. Das bedeutet konkret:

- Von Seiten der Erziehungswissenschaft sollte ein verstärkter Fokus auf die empirische Untersuchung der konkreten Nutzung, „Inbetriebnahme“ und pädagogischen Wirksamkeit alternativer Schulraummodelle gelegt werden - und dies gerade auch hinsichtlich der damit verbundenen Praktiken des Hervorbringens

6 Hierzu sowie zum zweifellos vorhandenen Potenzial der „Phase Null“, ebenfalls als Instrument der Schulentwicklung und Lehrerprofessionalisierung zu dienen, siehe ausführlicher Zenke (2018a). Zu darüber hinausgehenden Bemühungen zur Etablierung einer anschließenden, sich speziell auf den Bezug neu errichteter Schulgebäude konzentrierenden „Phase Zehn“ siehe ebd. (S. 120 f.) sowie Seydel (2016, S. 24). 
pädagogischer Räume im schulischen Alltag. Gleichzeitig sollte in historiographischer Perspektive das „Scheitern“ der deutschen Großraumschulbewegung der 1970er-Jahre genauer in den Blick genommen werden, um so - in der Zusammenschau von Geschichte und Gegenwart - fundiertere Aussagen treffen zu können über organisatorische, pädagogische, didaktische und raumbezogene Gelingenswie Misslingensbedingungen der occupation und inhabitation alternativer Schulraummodelle.

- Parallel dazu - und hierauf aufbauend - sollten von Seiten der Schulentwicklungsforschung Strategien entwickelt und evaluiert werden, unter Zuhilfenahme derer Einzelschulen gezielt darin unterstützt werden könnten, den Übergang in neue, möglicherweise deutlich offenere Räumlichkeiten bewusst als Anlass und Instrument der Schul- und Unterrichtsentwicklung zu nutzen - und zwar idealerweise in Kombination mit bereits etablierten Formen der Nutzerbeteiligung beim Planungsprozess von neuen Schulgebäuden (Stichwort „Phase Null“).

- Unterstützt werden sollten diese Bemühungen darüber hinaus schließlich von Seiten der Lehrerbildung durch eine systematische Förderung der „spatial literacy“ von Lehrer*innen: durch Maßnahmen also, die sowohl angehende als auch bereits aktive Lehrkräfte einerseits für die grundsätzliche pädagogische Bedeutung des Raumes in der Schule sensibilisieren und ihnen andererseits didaktische Mittel an die Hand geben, um guten Unterricht auch jenseits des traditionellen Klassenzimmer-Flur-Ensembles zu realisieren.

All diese Bemühungen jedoch werden letztlich nur dann auch tatsächlich Früchte tragen können, wenn darüber hinaus von Seiten der Bildungspolitik a) Mittel zur Verfügung gestellt werden, um die skizzierten Schulentwicklungsprozesse auch finanziell zu unterstützen, und b) im Rahmen der Ausschreibung und Beauftragung neuer Schulbauten größerer Mut auch zu solchen Entwürfen bewiesen wird, die sich stärker als bisher vom traditionellen Konzept der Klassenraum-Flur-Schule entfernen. Denn wenigstens dies zeigt bereits ein einfacher Spaziergang durch die europäische Schullandschaft (vom Lernhaus im Campus in Osterholz-Scharmbeck über das Ørestad-Gymnasium in Kopenhagen bis hin zur University of Cambridge Primary School in Großbritannien): Zumindest auf Seiten der Schularchitektur scheint es nicht an Konzepten und Visionen für eine auch baulich veränderte „Schule des 21. Jahrhunderts“ zu mangeln. Es ist daher also höchste Zeit, dass auch Erziehungswissenschaft, Schulentwicklung, Lehrerbildung und Politik sich noch einmal deutlich mutiger als bereits bisher der damit verbundenen Chancen und Herausforderungen annehmen. 
„Vom Klassenzimmer zur Lernlandschaft“? |

\section{Literatur und Internetquellen}

Acton, R., Riddle, M., \& Sellers, W. (2018). A Review of Post-Occupancy Evaluation Tools. In S. Alterator \& C. Deed (Hrsg.), School Space and Its Occupation. Conceptualising and Evaluating Innovative Learning Environments (Advances in Learning Environments Research) (S. 203-221). Boston, MA: Brill Sense.

Alterator, S., \& Deed, C. (2018). Framing Innovative Learning Environments. In S. Alterator \& C. Deed (Hrsg.), School Space and Its Occupation. Conceptualising and Evaluating Innovative Learning Environments (Advances in Learning Environments Research) (S. 3-11). Boston, MA: Brill Sense. https://doi.org/10.1163/9789004379664_001

Auer, T., \& Nagler, F. (2017). Zukunftsfähiger Schulbau: 12 Schulen im Vergleich. DBU Bauband, Bd. 2. Hrsg. von S. Djahanschah. München: Edition Detail. https://doi. org/10.11129/9783955533663

Berdelmann, K., Burri, L., Dinsleder, C., Johann, N., Kirchgässner, U., Laros, A., et al. (2016). Schularchitektur im Dialog: Fallstudie und Möglichkeitsräume. Bern: hep.

Berdelmann, K., \& Reh, S. (2015). Adressierung durch den Raum - (Lieblings-)Plätze in der Schule: Eine fotoethnographische Exploration. In T. Alkemeyer, H. Kalthoff \& M. Rieger-Ladich (Hrsg.), Bildungspraxis. Körper - Räume - Objekte (S. 183-205). Weilerswist: Velbrück Wissenschaft. https://doi.org/10.5771/9783845277349-183

Blömer, D. (2011). Topographie der Gesamtschule: Zum Zusammenhang von Raum und Pädagogik. Bad Heilbrunn: Klinkhardt.

Bradbeer, C. (2016). Working Together in the Space-Between: Pedagogy, Learning Environment and Teacher Collaboration. In W. Imms, B. Cleveland \& K. Fisher (Hrsg.), Evaluating Learning Environments. Snapshots of Emerging Issues, Methods and Knowledge (Advances in Learning Environments Research) (S. 75-90). Rotterdam: Sense Publishers. https://doi.org/10.5771/9783845277349-183

Breidenstein, G., \& Dorow, S. (2015). Arbeitsplätze: Beobachtungen und Analysen aus dem individualisierten Unterricht. In T. Alkemeyer, H. Kalthoff \& M. Rieger-Ladich (Hrsg.), Bildungspraxis. Körper - Räume - Objekte (S. 159-181). Weilerswist: Velbrück Wissenschaft. https://doi.org/10.5771/9783845277349-159

Breidenstein, G., \& Rademacher, S. (2017). Individualisierung und Kontrolle: Empirische Studien zum geöffneten Unterricht in der Grundschule (Studien zur Schul- und Bildungsforschung, Bd. 60). Wiesbaden: Springer Fachmedien. https://doi.org/10.1007/ 978-3-658-13805-9

Brokamp, B., \& Platte, A. (2011). Inklusive Schulen bauen! Erziehung \& Unterricht, (5/6), $452-460$.

Brost, M. (2015). Deutschland lässt seine Zukunft verrotten. Zugriff am 27.09.2019. Verfügbar unter: https://www.zeit.de/wirtschaft/2015-09/marode-schulen-bildungspolitik.

Bundesministerium der Finanzen (2019). Förderung von Investitionen finanzschwacher Kommunen. Pressemitteilung vom 02.04.2019. Zugriff am 27.09.2019. Verfügbar unter: https://www.bundesfinanzministerium.de/Content/DE/Standardartikel/Themen/Oef fentliche_Finanzen/Foederale_Finanzbeziehungen/Kommunalfinanzen/Kommunalin vestitionsfoerderungsfonds/Foerderung-von-Investitionen-finanzschwacher-Kommu nen.html.

Cleveland, B. (2016). Emerging Methods for the Evaluation of Physical Learning Environments. In W. Imms, B. Cleveland \& K. Fisher (Hrsg.), Evaluating Learning Environments. Snapshots of Emerging Issues, Methods and Knowledge (Advances in Learning Environments Research) (S. 93-105). Rotterdam: Sense Publishers. https:// doi.org/10.1007/978-94-6300-537-1_7 
Cleveland, B., \& Fisher, K. (2014). The Evaluation of Physical Learning Environments: a Critical Review of the Literature. Learning Environment Research, 17, 1-28. https://doi. org/10.1007/s10984-013-9149-3

Deed, C., \& Lesko, T. (2015). 'Unwalling' the Classroom: Teacher Reaction and Adaptation. Learning Environment Research, 18, 217-231. https://doi.org/10.1007/s10984-015-9181-6

Egger, J. (2019). Häuser machen Schule: Eine architektursoziologische Analyse gebauter Bildung (Rekonstruktive Bildungsforschung, Bd. 27). Wiesbaden: Springer Fachmedien. https://doi.org/10.1007/978-3-658-26653-0

Fisher, K. (2004). Revoicing Classrooms: a Spatial Manifesto. FORUM, 46 (1), 36-38. https://doi.org/10.2304/forum.2004.46.1.8

Gebhard, U., Hummrich, M., Rabenstein, K., \& Reh, S. (2015). Räume, Dinge und schulisches Wissen: Eine Einführung. Zeitschrift für interpretative Sozialforschung, 4, 3-14. https://doi.org/10.3224/zisu.v4i1.21311

Gislason, N. (2010). Architectural Design and the Learning Environment: A Framework for School Design Research. Learning Environment Research, 13, 127-145. https://doi. org/10.1007/s10984-010-9071-x

Gislason, N. (2018). The Whole School: Planning and Evaluating Innovative Middle and Secondary Schools. In S. Alterator \& C. Deed (Hrsg.), School Space and Its Occupation. Conceptualising and Evaluating Innovative Learning Environments (Advances in Learning Environments Research) (S. 187-201). Boston, MA: Brill Sense.

Glaser, E., Koller, H.-C., Thole, W., \& Krumme, S. (Hrsg.). (2018). Räume für Bildung - Räume der Bildung: Beiträge zum 25. Kongress der Deutschen Gesellschaft für Erziehungswissenschaft. Opladen, Berlin \& Toronto: Barbara Budrich. https://doi. org/10.2307/j.ctvdf0hv3.4; https://doi.org/10.2307/j.ctvdf0hv3.3

Göbel, H. K. (2016). Die atmosphärische Vermittlung der Moderne: Architektur und Gebäude in praxeologischer Perspektive. In H. Schäfer (Hrsg.), Praxistheorie. Ein soziologisches Forschungsprogramm (S. 199-220). Bielefeld: transcript.

Healy, S. (2016). Evaluating Spaces of Pedagogic Affect. In W. Imms, B. Cleveland \& K. Fisher (Hrsg.), Evaluating Learning Environments. Snapshots of Emerging Issues, Methods and Knowledge (Advances in Learning Environments Research) (S. 235-250). Rotterdam: Sense Publishers. https://doi.org/10.1007/978-94-6300-537-1_17

Hedtke, K. (2016). Sanierungsstau in Schulen: Eine Schande. Erziehung und Wissenschaft. Allgemeine Deutsche Lehrerzeitung, 68 (9), 6-10.

Huber, L., \& Thormann, E. (2002). Großraumschulen - Erwartungen und Erfahrungen. Oder: „Vom versuchsweisen Wegfall der Wände“. In L. Wigger \& N. Meder (Hrsg.), Raum und Räumlichkeit. Festschrift für Harm Paschen (S. 65-86). Bielefeld: Janus.

Imhäuser, K.-H. (2012). Inklusion und die Konsequenzen: Raumanforderungen an eine „Schule für alle“. In E. Rauscher (Hrsg.), Lernen und Raum. Gebaute Pädagogik und pädagogische Baustellen (Pädagogik für Niederösterreich) (S. 185-196). Baden: Pädagogische Hochschule Niederösterreich.

Imms, W. (2016). New Generation Learning Environments: How Can We Find out if What Works Is Working? In W. Imms, B. Cleveland \& K. Fisher (Hrsg.), Evaluating Learning Environments. Snapshots of Emerging Issues, Methods and Knowledge (Advances in Learning Environments Research) (S. 21-34). Rotterdam: Sense Publishers. https://doi. org/10.1007/978-94-6300-537-1

Imms, W. (2018). Innovative Learning Spaces: Catalysts/Agents for Change, or 'Just Another Fad'? In S. Alterator \& C. Deed (Hrsg.), School Space and Its Occupation. Conceptualising and Evaluating Innovative Learning Environments (Advances in Learning Environments Research) (S. 107-118). Boston, MA: Brill Sense.

Imms, W., Cleveland, B., \& Fisher, K. (2016). Pursuing That Elusive Evidence about What Works in Learning Environment Design. In W. Imms, B. Cleveland \& K. Fisher 
„Vom Klassenzimmer zur Lernlandschaft“? |

(Hrsg.), Evaluating Learning Environments. Snapshots of Emerging Issues, Methods and Knowledge (Advances in Learning Environments Research) (S. 3-16). Rotterdam: Sense Publishers. https://doi.org/10.1007/978-94-6300-537-1_1

Kahlert, J., Nitsche, K., \& Zierer, K. (Hrsg.). (2013). Räume zum Lernen und Lehren: Perspektiven einer zeitgemäßen Schulraumgestaltung. Bad Heilbrunn: Klinkhardt.

Kalthoff, H., Rieger-Ladich, M., \& Alkemeyer, T. (2015). Bildungspraxis - eine Einleitung. In T. Alkemeyer, H. Kalthoff \& M. Rieger-Ladich (Hrsg.), Bildungspraxis. Körper Räume - Objekte (S. 9-33). Weilerswist: Velbrück Wissenschaft. https://doi.org/10. 5771/9783845277349-9

Kariippanon, K. E., Cliff, D. P., Lancaster, S. L., Okely, A. D., \& Parrish, A.-M. (2018). Perceived Interplay between Flexible Learning Spaces and Teaching, Learning and Student Wellbeing. Learning Environment Research, 21, 301-320. https://doi.org/ 10.1007/s10984-017-9254-9

Kessl, F. (2016). Erziehungswissenschaftliche Forschung zu Raum und Räumlichkeit: Eine Verortung des Thementeils „Raum und Räumlichkeit in der erziehungswissenschaftlichen Forschung". Zeitschrift für Pädagogik, 62, 5-19.

Kricke, M., Reich, K., Schanz, L., \& Schneider, J. (2018). Raum und Inklusion: Neue Konzepte im Schulraum. Weinheim \& Basel: Beltz.

Lippman, P. C., \& Matthews, E. (2018). Re-Imagining the Open Classroom. In S. Alterator \& C. Deed (Hrsg.), School Space and Its Occupation. Conceptualising and Evaluating Innovative Learning Environments (Advances in Learning Environments Research) (S. 63-85). Boston, MA: Brill Sense.

Löw, M. (2012). Raumsoziologie (7. Aufl.). Frankfurt a. M.: Suhrkamp.

Montag Stiftung Jugend und Gesellschaft (Hrsg.). (2015). Fünfmal Phase Null: Dokumentation der Pilotprojekte „Schulen planen und bauen“. Bonn: Montag Stiftung.

Montag Stiftung Jugend und Gesellschaft (2017). Schulen planen und bauen 2.0: Grundlagen, Prozesse, Projekte. Berlin: jovis.

Montag Stiftung Urbane Räume, Montag Stiftung Jugend und Gesellschaft, Bund Deutscher Architekten \& Verband Bildung und Erziehung (2013). Leitlinien für leistungsfähige Schulbauten in Deutschland. Bonn \& Berlin: Eigenverlag.

Puls (o. J.). Puls+ Erasmus+ Projekt. Zugriff am 27.09.2019. Verfügbar unter: https://www. pulsnetz.org/forschungsprojekt.

Rabenstein, K., \& Reh, S. (2008). Über die Emergenz von Sinn in pädagogischen Praktiken: Möglichkeiten der Videographie im ,Offenen Unterricht'. In H.-C. Koller (Hrsg.), Sinnkonstruktion und Bildungsgang. Zur Bedeutung individueller Sinnzuschreibungen im Kontext schulischer Lehr-Lern-Prozesse. Opladen \& Farmington Hills, MI: Barbara Budrich. https://doi.org/10.2307/j.ctvdf06q4.10

Ramseger, J. (2017). Pädagogik durch Architektur ermöglichen. In Senatsverwaltung für Bildung, Jugend und Familie (Hrsg.), Berlin baut Bildung. Die Empfehlungen der Facharbeitsgruppe Schulraumqualität. Band 1: Bericht (S. 6). Berlin: SBJF.

Reh, S. (2011). Individualisierung und Öffentlichkeit: Lern-Räume und Subjektivationsprozesse im geöffneten Grundschulunterricht. In K. Amos, W. Meseth \& M. Proske (Hrsg.), Öffentliche Erziehung revisited. Erziehung, Politik und Gesellschaft im Diskurs (S. 33-52). Wiesbaden: VS. https://doi.org/10.1007/978-3-531-92615-5_2

Reh, S., \& Kolbe, F.-U. (2009). Grenzverschiebungen: Diskurse und Praktiken in Ganztagsschulen. In J. Böhme (Hrsg.), Schularchitektur im interdisziplinären Diskurs. Territorialisierungskrise und Gestaltungsperspektiven des schulischen Bildungsraums (S. 103-118). Wiesbaden: VS. https://doi.org/10.1007/978-3-531-91868-6_7

Reich, K. (2012). Zusätzlicher Raumbedarf: Inklusion und Ganztagsschule als Herausforderung auch für den Schulbau. Lernende Schule, 15 (59), 14-17. 
Reich, K. (2014). Inklusive Didaktik: Bausteine für eine inklusive Schule. Weinheim \& Basel: Beltz.

Schneider, J. (2012). Neue Schulen in alten Gebäuden? Wie sich Schulen durch Umbaumaßnahmen neuen pädagogischen Anforderungen stellen können. Lernende Schule, 15 (59), 8-13.

Schöning, W., \& Schmidtlein-Mauderer, C. (Hrsg.). (2015). Inklusion sucht Raum. Porträtierte Schulentwicklung. Bern: hep.

Schöning, W., \& Schmidtlein-Mauderer, C. (2016). Die Dimension des Raums in ihrer Bedeutung für die inklusive Schulentwicklung. In W. Schöning \& J. A. Fuchs (Hrsg.), Inklusion: Gefordert! Gefördert? Schultheoretische, raumtheoretische und didaktische Zugänge (S. 77-94). Bad Heilbrunn: Klinkhardt.

Schrittesser, I., Fraundorfer, A., \& Krainz-Dürr, M. (Hrsg.). (2012). Innovative Learning Environments. Fallstudien zu pädagogischen Innovationsprozessen. Wien: Facultas.

Seydel, O. (2016). Die Phase Null: Schulbauvorhaben gemeinsam planen. Grundschule, (7), 22-24.

Sigurðardóttir, A. K., \& Hjartarson, T. (2016). School Buildings and Classroom Environments in Iceland. In U. Stadler-Altmann (Hrsg.), Lernumgebungen. Erziehungswissenschaftliche Perspektiven auf Schulgebäude und Klassenzimmer (S. 31-47). Opladen, Berlin \& Toronto: Barbara Budrich. https://doi.org/10.2307/j.ctvbkk1r7.5

Stadler-Altmann, U. (2016a). Gebaute Umgebung als Lernumgebung: Haben Schulgebäude und Klassenzimmer Einfluss auf Lehren und Lernen? In U. Stadler-Altmann (Hrsg.), Lernumgebungen. Erziehungswissenschaftliche Perspektiven auf Schulgebäude und Klassenzimmer (S. 49-68). Opladen, Berlin \& Toronto: Barbara Budrich. https://doi. org/10.2307/j.ctvbkk1r7.6

Stadler-Altmann, U. (Hrsg.). (2016b). Lernumgebungen: Erziehungswissenschaftliche Perspektiven auf Schulgebäude und Klassenzimmer. Opladen, Berlin \& Toronto: Barbara Budrich. https://doi.org/10.2307/j.ctvbkk1r7

Stadler-Altmann, U. (2016c). Lernumgebungen: Erziehungswissenschaftliche und architekturkritische Perspektiven auf Schulgebäude und Klassenzimmer. In U. Stadler-Altmann (Hrsg.), Lernumgebungen. Erziehungswissenschaftliche Perspektiven auf Schulgebäude und Klassenzimmer (S. 7-16). Opladen, Berlin \& Toronto: Barbara Budrich. https://doi. org/10.2307/j.ctvbkk1r7.3

Wetzel, J. (2019). Das liegende Klassenzimmer. Süddeutsche Zeitung vom 29.04.2019, 22.

Windrath, M. (2019). Schulen in der Warteschleife. Bildungslandschaft: Elternvertreter enttäuscht wegen Verzögerung. Kölnische Rundschau vom 15.02.2019, 30.

Wood, A. (2018). The Politics of Post Occupancy Evaluation: The Example of Schools. In S. Alterator \& C. Deed (Hrsg.), School Space and Its Occupation. Conceptualising and Evaluating Innovative Learning Environments (Advances in Learning Environments Research) (S. 121-134). Boston, MA: Brill Sense.

Zenke, C. T. (2017). Schule als inklusiver Raum? Zeitschrift für Inklusion, (4). Zugriff am 27.09.2019. Verfügbar unter: https://www.inklusion-online.net/index.php/inklusiononline/article/view/441/342.

Zenke, C. T. (2018a). Raum für Veränderung: Schularchitektur als Ausgangspunkt und Instrument von Schulentwicklung. Transfer Forschung $<->$ Schule, 4, 112-123.

Zenke, C. T. (2018b). Schule gemeinsam gestalten. Multiprofessionelle Kooperation im Schulbau. Lernende Schule, (81), 25-27.

Zenke, C. T. (2019). Raumbezogene Schulentwicklung in einer inklusiven Schule: Zur Nutzungsgeschichte des Unterrichtsgroßraums der Laborschule Bielefeld. PFLB - PraxisForschungLehrer ${ }^{*}$ innenBildung, 1 (1), 20-41. https://doi.org/10.4119/pflb-3173

Zinner, M. (2014). schulRAUMkultur: Wie Anstalten loslassen? Wie in Schulen heimkommen? zeitschrift ästhetische bildung, 6 (1), 1-17. 
Zschiesche, B. (2019). Schulraum als Heimat: Eine empirische Studie zum Raumerleben von Grundschulkindern. Bad Heilbrunn: Klinkhardt.

Christian Timo Zenke, Dr. phil., geb. 1979, Akademischer Rat (a.Z.) in der Fakultät für Erziehungswissenschaft der Universität Bielefeld.

Korrespondenzadresse: Universität Bielefeld, Fakultät für Erziehungswissenschaft, Universitätsstraße 25, 33615 Bielefeld

E-Mail: timo.zenke@uni-bielefeld.de 